Meta

Journal des tradlucteurs

Translators' Journal

\title{
La documentation de base en matière de langue française
}

\section{Madeleine Sauvé}

Volume 25, numéro 1, mars 1980

La documentation

URI : https://id.erudit.org/iderudit/004033ar

DOI : https://doi.org/10.7202/004033ar

Aller au sommaire du numéro

\section{Éditeur(s)}

Les Presses de l'Université de Montréal

\section{ISSN}

0026-0452 (imprimé)

1492-1421 (numérique)

Découvrir la revue

Citer cet article

Sauvé, M. (1980). La documentation de base en matière de langue française. Meta, 25(1), 87-100. https://doi.org/10.7202/004033ar d'utilisation que vous pouvez consulter en ligne.

https://apropos.erudit.org/fr/usagers/politique-dutilisation/ 


\title{
La documentation de base en matière de langue française
}

\author{
Madeleine Sauvé \\ grammairien,
}

Université de Montréal

Un titre aussi général appelle une double précision. Sous l'appellation : 《la documentation de base », on entend «les ouvrages de référence indispensables »; sous l'expression : « en matière de langue française », il faut lire : « en ce qui concerne la production d'énoncés ou la rédaction de textes en français ».

Il s'agit donc d'un propos éminemment pragmatique conçu en fonction des besoins des rédacteurs, des réviseurs, des traducteurs, des conseillers linguistiques ou, d'une façon plus générale, des usagers d'un service de documentation destiné à ces diverses catégories de personnel. Ce propos, quelque peu ambitieux en soit, reprendra au fil du texte des proportions modestes du fait que les considérations exposées feront nécessairement référence à l'expérience limitée de la signataire du présent article.

Dans cette perspective, on peut répartir comme suit les ouvrages de référence indispensables en matière de langue française :

1. les dictionnaires de langue et les dictionnaires encyclopédiques;

2. les dictionnaires spécifiques : dictionnaire étymologique, dictionnaire analogique, dictionnaire des synonymes, etc. ;

3. les grammaires et les ouvrages similaires.

\section{LES DICTIONNAIRES DE LANGUE ET LES DICTIONNAIRES ENCYCLOPÉDIQUES}

\subsection{Les dictionnaires de langue}

\subsubsection{Le Dictionnaire de l'Académie et le Littré}

Parlant de dictionnaires de langue, il faut d'abord reconnaître que le dictionnaire officiel de la langue française est celui de l'Académie, mais force nous est de constater que le rythme de publication de cet ouvrage aussi bien que la politique qui anime ses auteurs font que le Dictionnaire de l'Académie est toujours un instrument de travail incomplet ${ }^{1}$ : on ne saurait efficacement s'y reporter dans la pratique quotidienne. Il reste que, dans certains cas, il sera opportun, sinon nécessaire, de consulter l'Académie ${ }^{2}$.

1. Les deux dernières rééditions du Dictionnaire de l'Académie remontent à $1878(7 \mathrm{e})$ et

à 1931-1935 (8e).

2. A l'occasion de la polémique relative au mot stop, par exemple, il était intéressant de constater que la célèbre institution avait accueilli le mot stopper cent ans plus tôt, soit dans la septième édition de son dictionnaire (1878). 
Il faut également considérer à part le grand dictionnaire de la fin du XIX siècle qu'est le Littré. Composé et publié entre 1846 et 1872 , à l'exception du Supplément qui date de 1877 , ce dictionnaire a été réédité intégralement depuis peu. Malgré le fait qu'il ne soit plus à jour, le Littré demeure un instrument de travail utile qui supporte la comparaison avec les autres grands dictionnaires de langue parus depuis une vingtaine d'années.

Dans le texte serré de ses sept volumes, le Littrés présente une riche collection d'exemples empruntés aux écrivains du XvIr ${ }^{e}$, du Xvmir et de la première moitié du XIX ${ }^{e}$ siècle; il offre d'intéressantes remarques grammaticales et stylistiques ; enfin, il donne d'utiles renseignements d'ordre historique et étymologique.

Les deux autres "grands " » que l'on doit obligatoirement voir figurer sur les rayons du service de documentation qui nous intéresse actuellement sont le Grand Robert et le Grand Larousse de la langue française.

\subsubsection{Le Grand Robert}

Que nous présente le grand Dictionnaire alphabétique et analogique de la langue française ${ }^{5}$ de $\mathrm{P}$. Robert? Soucieux de l'usage contemporain, l'auteur reprend et poursuit l'œuvre de dépouillement accomplie par É. Littré ; ainsi, * tout en conservant aux créateurs de la langue classique la place éminente qui leur appartient sans conteste ${ }^{6} \gg, P$. Robert puise abondamment, pour faire l'inventaire des mots et de leurs significations, dans les œuvres des cent dernières années?

Mais l'originalité de ce dictionnaire de langue tient à la conception qui préside à l'agencement de son contenu. À la fois alphabétique et analogique, le Grand Robert rend immédiatement accessible à l'utilisateur une vaste et précieuse information du fait que chaque mot traité est mis en rapport avec les autres mots que celui-ci évoque logiquement. Ce procédé permet de passer du connu à l'inconnu, de l'idée vague à l'expression précise, du vocabulaire commun à des vocabulaires particuliers.

Si le dictionnaire Robert recèle une richesse propre du fait qu'il tire large profit des ressources de l'analogie, c'est-à-dire des liens qu'il établit entre les choses et des rapprochements d'idées qu'il suggère, il n'en reste pas moins un dictionnaire alphabétique. Conséquemment, ce sont les termes mêmes des défini-

3. E. Littré, Dictionnaire de la langue française, édition intégrale, Paris, GallimardHachette, 1971-1975.

4. Il serait juste de nommer également ici le Dictionnaire général de la langue française de A. Hatzfeld et A. Darmesteter, avec le concours de A. Thomas. - La contribution la plus remarquable de ce dictionnaire de langue rédigé au dernier quart du xixe siècle et dont la réédition intégrale (2 vol.) a été faite par Delagrave en 1964, est d'ordre étymologique et sémantique. Le Traité de la formation de la langue, de près de trois cents pages, qui précède le dictionnaire proprement dit expose l'histoire des mots français et les règles de leur formation.

5. P. Robert, Dictionnaire alphabétique et analogique de la langue française, Paris, Société : du Nouveau Littré, 1953-1964, 6 vol.; Supplément, 1970.

6. P. Robert, ibid., tome premier, «Introduction 》, p. IV.

7. Compte tenu de la date de la publication du Grand Robert, il faut comprendre que, par rapport au Littré, le dépouillement s'enrichit de citations empruntées aux cuvres publiées entre 1860 et 1960 environ. 
tions qui servent de structure à l'ensemble de l'œuvre : aussi, présenter clairement le sens des mots et des expressions est-il le souci premier de l'auteur ${ }^{8}$.

De ce fait, le Robert est un guide pour le locuteur soucieux d'exprimer adéquatement sa pensée en tenant compte à la fois des exigences de la propriété des termes et des nuances traduites par chacun d'eux. $\AA$ titre d'illustration, considérons l'article consacré au mot Certitude : d'emblée, on y décèle deux acceptions majeures du mot. On a, d'une part : "Caractère de ce qui est certain », c'est la certitude entendue objectivement; et, d'autre part : "Assurance pleine et entière que l'on a d'une chose », c'est la certitude subjective. De ces définitions, on est renvoyé, dans le premier cas, à "Evidence, vérité, sûreté 》; dans le second, à : "Conviction, croyance, opinion 》. Etre attentif à ces données, c'est éviter d'employer sans contrôle ni vérification le premier mot qui vient sous la plume, c'est développer à la fois la justesse de l'expression et la rigueur de la pensée.

Signalons en outre que le Robert rend d'utiles services en faisant souvent précéder d'une marque d'usage les définitions présentées; on peut ainsi savoir immédiatement que tel mot ou tel emploi est argotique, populaire ou régional, ou bien qu'il est didactique ou littéraire, ou encore qu'il appartient au langage scientifique ou technique.

La description sommaire des ressources du Robert serait incomplète si l'on ne rappelait qu'aux six volumes publiés jusqu'à 1964 est venu s'ajouter, en 1970, un septième volume qui constitue le Supplément. Le secrétaire général de la rédaction, M. Alain Rey, fait remarquer que ce Supplément «ne constitue pas seulement une mise à jour, mais un élargissement des zones lexicales décrites dans les six premiers volumes». "Nous pensons, précise-t-il, qu'en 1970, le Robert présente plus fidèlement que jamais le lexique français en expansion ${ }^{\circ} . »$

Comme le chercheur a toujours intérêt et avantage à consulter plus d'une source, il en va de même pour le spécialiste de la langue. Il devra trouver sur le même rayon que le Grand Robert cet autre dictionnaire de langue qu'est le Grand Larousse de la langue française.

\subsubsection{Le Grand Larousse de la langue française (G.L.L.F.)}

Que réserve à l'utilisateur le Grand Larousse de la langue française ${ }^{10} ? \mathrm{Du}$ point de vue du lexique recensé, le G.L.L.F. comporte plus de 70000 mots ${ }^{11}$. La Préface du premier volume nous décrit comme suit l'intention des auteurs de ce dictionnaire : il « offre un instrument de connaissance du lexique de notre langue, dont la devise pourrait être : «Tous les aspects de la langue, et rien que la

8. Les associations d'idées, précédées de la lettre V. (Voir) et imprimées en caractères gras «se distinguent nettement dans le texte de ce qui appartient proprement à la définition. Les exemples illustrant les définitions sont en italique. Aucune confusion ne doit done obscurcir le sens des mots 》. (P. Robert, op. cit., tome premier, «Introduction $\gg$, p. VI.)

9. A. Rey, "Présentation du supplément ", dans P. Robert, ibid., Supplément, p. XIII.

10. Grand Larousse de la langue francaise, Paris, Larousse, 1971-1978, 7 vol.

11. Dossier spécialement conçu pour tous les enseignants de la langue française : Grand Larousse de la langue française, Paris, Larousse, s.d., p. 7. 
langue $» .[. .$.$] Il contient une description complète du lexique, selon la technique$ lexicographique mise au point depuis plus d'un siècle dans la collection des Larousse, mais aussi selon les données de la lexicologie moderne ${ }^{12} \gg$. C'est dire que cet ouvrage comporte non seulement le vocabulaire commun de la langue parlée et écrite, mais encore un grand nombre de termes non courants : termes de linguistique, de rhétorique et de grammaire; termes techniques et scientifiques nécessaires pour lire l'essentiel des textes spécialisés; termes de la langue classique ; termes de civilisation et termes historiques ; enfin, termes archaïques.

Certains titres de cette nomenclature appellent une explication. Dans la catégorie des termes techniques et scientifiques, le G.L.L.F. intègre largement les mots qui relèvent des sciences humaines : psychologie, psychiatrie, médecine, sociologie, économie, philosophie, etc. Il ne reprend cependant pas tous les termes techniques consignés dans le Grand Larousse encyclopédique dont il sera question ci-après; sauf exceptions, il laisse de côté les termes ayant rapport à des techniques anciennes ou les mots les plus spéciaux du vocabulaire des sciences exactes.

Bien qu'il soit essentiellement un dictionnaire de la langue du $\mathrm{XIX}^{\mathrm{e}}$ et du $x^{e}$ siècle, le G.L.L.F. ne s'interdit pas « des incursions dans le lexique de la langue classique des $\mathrm{XVII}^{\mathrm{e}}$ et $\mathrm{XVIII}^{\mathrm{e}}$ siècles ${ }^{13} \gg$. De ce fait, l'ouvrage offre des comparaisons utiles entre les emplois modernes et les emplois classiques de mots anciens qui ont pris aujourd'hui un sens nouveau (Ex. : Autoriser, employé au sens classique pour signifier «investir quelqu'un d'une autorité », et au sens moderne, pour dire : «donner la permission à quelqu'un »). D'aucuns regrettent cependant que l'abréviation class., qu'on lit spontanément comme classique, au sens d'usuel, s'applique généralement à des mots ou à des sens désuets.

Il y a également lieu d'indiquer que les auteurs du G.L.L.F., optant pour une description objective du lexique français contemporain, ont admis les termes 《contestés selon une vision puriste et puritaine de la langue ${ }^{14}$ ». De même, ils n'ont pas hésité à enregistrer un grand nombre de néologismes, le critère déterminant à cet égard étant celui « d'une certaine fréquence dans la presse ${ }^{15} 》$. Cette politique a aussi fait accueillir des termes étrangers et donner place aux termes familiers et argotiques.

Corrélativement, le G.L.L.F. offre à l'utilisateur les indications nécessaires pour lui permettre de donner à chaque mot ou à chaque emploi sa valeur de communication exacte. Il devient ainsi un instrument précieux pour le locuteurrédacteur qui veut $s^{\prime}$ exprimer avec justesse et correction. De plus, sans tenir lieu de grammaire normative, le G.L.L.F. peut, à plus d'un titre, servir d'ouvrage de référence à qui se pose des questions d'usage : Peut-on employer tel mot dans tel cas ? A quelles conditions l'emploi est-il correct? C'est surtout dans les articles

\footnotetext{
12. L. Guilbert, R. Lagane, G. Niobey, "Préface », dans Grand Larousse de la langue française, tome premier, 1971, p. I.

13. Ibid.

14. Dossier spécialement conçu pour tous les enseignants de la langue française : Grand Larousse de la langue française, p. 9.

15. Ibid.
} 
consacrés à ce qu'on appelle les mots «grammaticaux 》: prépositions, conjonctions, pronoms, etc., qu'il offre de telles indications.

À titre d'exemple, on verra comment l'article consacré à la préposition $\lambda$ est structuré selon les fonctions syntaxiques : I. $\dot{A}$ introduit les compléments circonstanciels du verbe. II. $\dot{A}$ marque l'appartenance. III. $\dot{A}$ introduit le complément d'objet d'un verbe. IV. $\dot{A}$ introduit le complément d'un adjectif. V. Emplois divers. On trouvera évidemment des titres similaires dans l'article traitant de la préposition $D e$. En voilà assez pour trancher bien des cas d'hésitation relatifs à l'emploi de ces prépositions dans tel ou tel contexte. Cet aspect didactique est encore marqué du fait que le G.L.L.F. inclut des remarques à la suite des termes dont l'emploi soulève quelques difficultés grammaticales. Ainsi on relève neuf remarques de ce type pour la seule préposition $A$.

Par ailleurs, le G.L.L.F. contient une véritable encyclopédie de grammaire et cle linguistique répartie en cent soixante-neuf articles qui trouvent place à leur ordre alphabétique (Ex. : L'attribut; Les synonymes; Le verbe ; etc.). L'introduction à cette partie du dictionnaire décrit comme suit le propos général des auteurs en cette matière : faire goûter au grand public «l'intérêt d'une grammaire qui soit plus une peinture qu'un code ${ }^{16} \gg$; il va de soi que linguistes et philosophes $y$ trouveront une documentation intéressante sur les diverses notions traitées.

Enfin, considérant que le dictionnaire de langue a essentiellement trait au lexique, on terminera l'exposé des considérations sur le G.L.L.F. en attirant l'attention sur le fait que les auteurs ont cru bon de joindre au premier volume de l'ouvrage une imposante étude de soixante-dix pages sur le système de la création lexicale dans la langue française. Ces «fondements lexicologiques du dictionnaire » apportent en la matière l'éclairage de la linguistique moderne qui considère le lexique comme étroitement lié, dans sa formation même, à la structure profonde de l'énoncé.

\subsubsection{Le Trésor de la langue française (T.L.F.)}

Parmi les grands dictionnaires de langue, on fera une place à part au Trésor de la langue française. Dictionnaire de la langue du $19^{\circ}$ et du $20^{\circ}$ siècle (17891960). Publié sous la direction de Paul Imbs, de l'Institut, et édité par le Centre national de la recherche scientifique, ce trésor ne nous a encore révélé qu'une mince partie de ses richesses : les six premiers tomes actuellement disponibles comprennent les entrées de $A$ à $D E S$.

Dictionnaire de la langue écrite du dix-neuvième et du vingtième siècle (jusqu'à 1964 environ), le T.L.F. atteint son objectif : l'ensemble des textes dépouillés pour fournir la nomenclature et les exemples est de loin le plus important qui ait été mis en œuvre jusqu'ici. Ce corpus est composé d'une double série de textes : a) des textes littéraires choisis en raison de leur valeur de forme et de contenu ; b) des textes non littéraires choisis comme témoins autorisés, d'une part, d'une langue commune soignée, d'autre part, d'un minimum de vocabulaire

16. H. Bonnard, «Introduction à la partie «grammaire et linguistique », dans Grand Larousse de la langue française, tome premier, 1971 , p. VI. 
spécial usité dans les domaines où se sont exprimées la pensée et l'activité française durant ces deux siècles ${ }^{17}$.

La nature et le nombre des textes choisis indique le type de langue visé par les auteurs du T.L.F. : il s'agit de « la langue d'une culture à dominante humaniste, écrit $P$. Imbs, privilégiant donc l'expression littéraire, mais incluant déjà une certaine dose de vocabulaire spécial, imposé à la "littérature » même par l'évolution d'une société de plus en plus marquée par la pensée scientifique et par les activités techniques de plus en plus différenciées ${ }^{18}$ ».

Du point de vue de la nomenclature générale, le T.L.F. équivaut à peu près aux deux «grands » dont on vient de parler ${ }^{19}$; de l'analyse du contenu du corpus dépouillé, il se dégage que la place accordée aux mots usuels et aux mots techniques est respectivement de $80 \%$ et de $20 \%$. Point n'est besoin de dire que le T.L.F. l'emporte sur les deux autres dans le traitement des mots : en règle générale, il réserve aux mots usuels trois à quatre fois plus d'espace que ses concurrents.

Si la nomenclature du Trésor est large, son mérite majeur réside «dans la quantité, la qualité, l'organisation de ce discours sur le mot-nommant qu'on attend avant tout, et à peu près uniquement, d'un dictionnaire de langue ${ }^{20} \%$. La brièveté des définitions tient également de ce caractère : l'abondance des exemples et la variété des citations font apparaître les valeurs et les nuances des mots mieux que ne le ferait une longue définition. Par ailleurs, des notices phonétiques, historiques et étymologiques situent le mot dans une large perspective qui permet de respecter le fait que le sens moderne d'un mot est chargé de ses sens anciens.

Les articles consacrés aux préfixes et aux suffixes ou aux éléments préfixaux et suffixaux rappellent fort utilement au lecteur les normes de formation des mots qui respectent le système de la langue, tout en promouvant la souplesse du vocabulaire pour répondre au besoin sans cesse croissant de mots nouveaux.

$\mathrm{Du}$ point de vue grammatical, l'apport du T.L.F. est moins important que celui du G.L.L.F.; plus orienté vers la sémantique et la linguistique, le T.L.F. présente cependant de bonnes notices au fil de sa nomenclature alphabétique : identification des parties du discours, construction des verbes, emplois absolus, etc., voilà autant d'indications de nature grammaticale qui permettent de construire de bons énoncés. De livre consulté en cas d'embarras de lecture, le T.L.F. devient ainsi, à sa manière, un auxiliaire de qui veut dire ou écrire correctement et bellement sa langue.

Il faut bien sûr regretter de devoir attendre au moins une dizaine d'années encore avant de disposer de l'ensemble du Dictionnaire de la langue du $19^{\circ}$ et du

17. Voir P. Imbs, «Le dictionnaire T.L.F. : Cahier de normes rédactionnelles 》, supplément $n^{\circ} 4$ de la revue Le Français moderne, Trésor de la langue française, Nancy, fascicule 4,1977 , p. $49-60$.

18. Ibid., p. 51-52.

19. Un large vocabulaire technique, rassemblé sous des rubriques étymologiques (Ex. : anthropo-, archéo-, chrono-) fera cependant du T.L.F. terminé un dictionnaire dont les entrées pourraient égaler celles des grands dictionnaires encyclopédiques tels que le Larousse ou le Quillet.

20. J. Cellard, \&Le trésor de la langue française », dans Le Monde, 18 février 1972, p. 20. 
$20^{\circ}$ siècle. Les six volumes disponibles n'en doivent pas moins figurer en bonne place sur les rayons réservés aux grands dictionnaires de langue.

\subsubsection{Le Petit Robert, le Dictionnaire du français contemporain (D.F.C.) et le Lexis}

Les grands dictionnaires de langue sont indispensables; les dictionnaires de langue en un volume ont aussi leur place dans un centre de documentation qui veut mettre à la disposition des utilisateurs les outils de base en matière de français. Plus maniables, plus commodes, les usuels présentent en outre l'avantage d'être régulièrement mis à jour.

Les trois noms mentionnés en rubrique indiquent un choix, bien sûr, mais ne prononcent pas l'exclusive contre les autres dictionnaires de même format. Rappelons à cet égard que c'est principalement, sinon exclusivement, l'expérience quotidienne de la signataire du présent article qui sous-tend les propos exprimés ici.

Sans dénier donc le mérite des « autres », on réserve une place de choix au Dictionnaire alphabétique et analogique de la langue française de P. Robert, plus commodément appelé le Petit Robert $I$ ou simplement le Petit Robert ${ }^{21}$.

L'éloge de cet excellent instrument de travail n'est plus à faire. Considérablement augmenté, le Petit Robert 1977 fait place à un grand nombre de nouveautés aussi bien du domaine technique et scientifique que de la langue familière.

Du point de vue de la conception, de l'esprit et de la méthode, il s'apparente au Grand Robert : il est descriptif, historique et analogique. Tout en conservant l'essentiel des qualités du Grand Robert, il le surpasse même à plus d'un titre ; en particulier, il s'enrichit des apports récents de la linguistique et il porte la marque de la contribution de spécialistes dans les domaines les plus diversifiés : phonétique, mathématiques, informatique, chimie, biologie, droit, sciences humaines, etc.

Malgré les limites imposées par le format réduit de ce type d'ouvrage, le Petit Robert complète chaque définition de notes étymologiques et de datations; de plus, sous chaque mot, sont donnés, outre ses divers sens, les locutions et les gallicismes où il entre, les indications grammaticales utiles, les marques d'usage et le domaine spécial auquel il appartient.

Modestement baptisé «petit», ce Robert condensé est en soi une mine témoignant de la richesse de la langue et constituant pour l'utilisateur un lieu qu'il n'a jamais fini d'explorer.

A titre d'appoint, il sera utile de trouver au rayon des dictionnaires de langue en un volume, le Dictionnaire du français contemporain ${ }^{22}$, d'une part, et le Lexis $^{23}$, d'autre part.

21. P. Robert, Dictionnaire alphabétique et analogique de la langue française, Le Petit Robert, Paris, Société du Nouveau Littré, Nouvelle édition, 1977, XXXI-2172 p.

22. Dictionnaire du français contemporain, Paris, Larousse, 1971, XXII-1224 p.

23. Larousse de la langue française Lexis, Paris, Larousse, 1977, XVI-2013 p. 
Edition conçue pour l'enseignement du français, le Dictionnaire du français contemporain comporte un vocabulaire de 25000 mots environ. Tenant compte des principes de la lexicologie moderne, il a pour objectif essentiel d'informer le locuteur-rédacteur sur les conditions d'insertion correcte du terme dans un énoncé. Pour ce faire, il indique les rapports de signification que le mot considéré entretient avec les termes de la phrase et avec les autres termes du lexique avec lesquels il partage une zone de signification commune. Il permet ainsi d'acquérir les éléments lexicaux de la structure fondamentale de la langue, et conséquemment, il aide à parler et à écrire conformément à la grammaire et avec les nuances nécessaires d'expression selon les situations.

Envisagé sous l'optique qui nous intéresse, le fait que les composés et les dérivés soient rattachés au mot simple ne fait pas de difficulté. Par ailleurs, le dégroupement qui consiste à présenter un même mot sous des rubriques différentes selon les champs de signification qu'il occupe, comporte des avantages méthodologiques indéniables.

Il faut sans doute regretter que le D.F.C. ne fasse aucune mention de l'étymologie ni de l'historique des mots et qu'il accorde volontiers la première place au sens actuel même lorsque ce sens est, de fait, second. Une telle option peut sans doute se justifier dans un manuel axé sur le français contemporain.

Signalons encore que les auteurs distinguent utilement les divers niveaux de langue, mais qu'ils se montrent très libéraux du point de vue du vocabulaire et de la syntaxe. Ainsi, après avoir entériné ce que d'aucuns considèrent comme une impropriété ou une incorrection, ils se bornent à mentionner entre parenthèses : «Emploi déconseillé par quelques grammairiens» (Ex. : Débuter une séance).

Ces réserves étant faites, on retiendra que le D.F.C. est un ouvrage de consultation facile, de présentation claire, de typographie aérée, somme toute un dictionnaire qu'il est utile d'avoir sous la main pour résoudre rapidement des problèmes courants d'écriture.

Du Larousse de la langue française en un volume, communément appelé Lexis, on indiquera d'abord, selon les termes mêmes de la Préface, qu'« il s'adresse à tous ceux qui veulent comprendre le fonctionnement de la langue et ont à se perfectionner dans sa pratique, à ceux qui doivent en acquérir la maîtrise intelligente et les techniques d'expression ${ }^{24}$ ».

Sa nomenclature de plus de 70000 mots reprend comme noyau de base les 25000 termes décrits dans le D.F.C., c'est-à-dire « ceux qui entrent dans l'usage écrit et parlé du français le plus habituel ». On y fait aussi bonne place aux néologismes « pourvu qu'ils aient un champ d'application suffisamment large ${ }^{25} \gg$; le vocabulaire des sciences et des techniques, en particulier, y est considérable.

Signalons encore que la méthode du regroupement et du dégroupement, préconisée dans le D.F.C., y est systématiquement utilisée : cette méthode a pour principal objectif de présenter dans chaque article une unité sémantique cohérente.

24. Larousse de la langue française Lexis, op. cit., « Préface», p. VI.

25. Ibid., « Préface », p. VII. 
Enfin, au fil des articles, le Lexis présente d'utiles renseignements orthographiques et grammaticaux que complètent avantageusement des renvois au Dictionnaire grammatical de plus de cinquante pages annexé à la description du lexique.

\subsection{Les dictionnaires encyclopédiques}

Si les ouvrages de référence indispensables en matière de langue française sont les dictionnaires de langue dont on vient de traiter abondamment, il reste que l'habitué d'un centre de documentation doit aussi pouvoir y consulter des dictionnaires encyclopédiques.

Pour distinguer sommairement le dictionnaire de langue du dictionnaire encyclopédique, on peut dire que le premier vise le sens alors que le second vise la réalité non linguistique. Le premier va au oceur des mots, le second cherche à découvrir le cour des choses. Le premier aide l'utilisateur à interpréter correctement ce qu'il entend ou ce qu'il lit et à exprimer avec justesse ce qu'il veut dire ou écrire ; le second se propose d'aider l'utilisateur non seulement à interpréter et à dire, mais encore et surtout à connaître et à manier les concepts et les choses. En général, le dictionnaire encyclopédique implique le dictionnaire de langue, mais celui-ci n'implique pas celui-là. Au demeurant, la distinction n'est pas toujours nette entre un dictionnaire de langue et une encyclopédie alphabétique.

Le Grand Larousse encyclopédique ${ }^{26}$ (G.L.E.) est un ouvrage particulièrement utile d'autant plus qu'il « joint au développement encyclopédique une étude sémantique, grammaticale et littéraire de chaque mot, appuyée par d'abondantes citations d'auteurs classiques ou contemporains ${ }^{27} \gg$. Avec ses dix volumes, publiés entre 1960 et 1964, et ses deux suppléments, qui datent respectivement de 1968 et de 1975, il comporte plus de 160000 articles traitant des domaines de connaissance les plus divers : agriculture, beaux-arts, droit, économie politique, géographie, histoire, littérature, musique, philosophie, spectacles, sports, sciences naturelles, sciences appliquées, sciences biologiques et médicales, etc.

Les suppléments comportent non seulement une mise à jour de la nomenclature, mais des ajouts considérables dans les domaines particuliers de l'informatique, des mathématiques modernes et de certaines sciences humaines : linguistique, écologie, psychologie, psychanalyse, anthropologie sociale, etc.

Pour apprécier le G.L.E. à sa juste valeur, il faut tenir compte du fait qu'il présente des articles fort détaillés sous des rubriques générales, de sorte que le mot technique utile se trouvera souvent dans le corps d'un article. Cet inconvénient réel est compensé par le fait que la lecture des articles permet généralement de faire surgir à l'esprit ou à la mémoire une quantité de termes spécialisés utiles.

Une autre encyclopédie peut, à certains titres, faire une concurrence serrée au G.L.E., c'est le Dictionnaire encyclopédique Quillet ${ }^{28}$. Dans les limites d'une

26. Grand Larousse encyclopédique, Paris, Larousse, 1960-1964, 10 vol.; Supplément, 1968 ; Deuxième supplément, 1975.

27. Ibid., tome premier, «Préface 》 (p. II).

28. Dictionnaire encyclopédique Quillet, Paris, Quillet, 1968-1970, 10 vol.; Supplément, 1971. 
expérience personnelle de consultation de cet ouvrage, nous y avons remarqué que le traitement de l'information en matière technique et scientifique y est plus poussé que dans le G.L.E.

Dans la section des dictionnaires encyclopédiques, on pourrait encore nommer le Larousse en couleurs ${ }^{29}$ réédité en 1976. Le lecteur s'y reportera volontiers pour la documentation visuelle que cet ouvrage propose : illustrations et schémas y sont généralement d'une remarquable qualité.

L'un des derniers-nés de la famille des Larousse encyclopédiques mérite d'être mentionné ici, c'est le nouvel Omnis ${ }^{30}$ qui forme avec le Lexis, le tandem " mots-choses ». Comme ce dernier, il offre, entre autres, l'avantage d'un maniement facile et d'une mise à jour plus récente ${ }^{31}$ que les grandes encyclopédies àlphabétiques.

Auxiliaires du locuteur-rédacteur, les dictionnaires encyclopédiques ne sauraient évidemment donner le dernier mot de l'information dans les divers domaines de la connaissance. Seuls les dictionnaires spécialisés et les études monographiques atteignent un tel objectif; il reste que des ouvrages tels que le Grand Larousse encyclopédique et le Dictionnaire encyclopédique Quillet, en particulier, peuvent répondre de façon adéquate aux besoins courants et devenir, à l'occasion, soit des introductions aux glossaires, soit des compléments utiles de ceux-ci.

$\mathrm{Au}$ terme de cette présentation des dictionnaires de langue et des dictionnaires encyclopédiques considérés comme indispensables - selon la problématique définie au départ - on pourra se demander comment, dans la pratique quotidienne, il conviendra de choisir l'un ou l'autre.

La réponse à cette interrogation n'est pas simple. A maintes occasions, il faudra non pas choisir l'un ou l'autre, mais consulter les uns et les autres : pour se convaincre de cette nécessité, il suffit d'observer les différences qui existent entre des dictionnaires de même type. $\AA$ cet égard, entrent en ligne de compte, non seulement les dates de rédaction, mais encore la conception différente des auteurs concernant l'usage des mots.

Par ailleurs, il est essentiel que chacun connaisse les ressources propres des instruments de référence mis à sa disposition de façon à savoir quasi spontanément qu'il trouvera réponse à telle difficulté dans le Lexis plutôt que dans l'Omnis, dans le Larousse plutôt que dans le Quillet, dans le Dictionnaire alphabétique et analogique plutôt que dạs le Grand Larousse de la langue française, ou vice verisa. Est-il besoin de signaler que les introductions, les préfaces et les avant-propos de ces ouvrages ouvrent des pistes à qui veut connaître leur contenu; de même la consultation de critiques autorisés offre, à l'occasion, des avantages réels, mais rien ne remplace la fréquentation patiente et méthodique d'un instrument de travail. Aussi faut-il convenir qu'en dernière analyse, cette

29. Paris, Larousse, 1976, 3 vol.

30. Encyclopédie alphabétique Larousse Omnis, Paris, Larousse, 1977, VII-1983 p.

31. A ce titre, un centre de documentation pourra juger opportun de mettre à la disposition des intéressés le Petit Larousse illustré ; enrichi de la section des noms propres, remis à jour annuellement, cet usuel est un témoin des « acceptions et expressions nouvelles » en circulation sur la place publique. 
connaissance de «ses» sources aussi bien que leur exploitation maximale ne s'acquerra que par voie de comparaison entre elles : c'est en procédant par additions, par recoupements, par retouches, qu'on arrive à maîtriser quelque peu les mots et à reconstituer, au terme de la démarche, l'énoncé le plus juste, le plus cohérent et le plus riche ou, tout au moins, le plus satisfaisant.

\section{L.ES DICTIONNAIRES SPÉCIFIQUES}

On a annoncé sous ce titre les ouvrages suivants : dictionnaire étymologique, dictionnaire analogique, dictionnaire des synonymes, etc.

Il s'agit en somme d'auxiliaires des dictionnaires de langue; aussi, compte tenu de l'optique présente aussi bien que des longs développements consacrés aux dictionnaires de langue, nous suffira-t-il de donner une nomenclature de ces ouvrages. Tout au plus, s'imposera-t-il d'ajouter à l'occasion une précision jugée utile au sujet de l'un ou de l'autre.

Pour l'étymologie, on aura le choix entre le Nouveau dictionnaire étymologique et historique de A. Dauzat, J. Dubois et $\mathrm{H}$. Mitterand ${ }^{32}$ et le Dictionnaire étymologique de la langue française de $\mathrm{O}$. Bloch et $\mathrm{W}$. van Wartburg ${ }^{33}$, à moins que l'on trouve un plus grand profit à consulter les grands dictionnaires de langue, ce qui est indéniablement le cas lorsqu'il s'agit des mots traités dans les six prenniers volumes du Trésor de la langue française.

Le Dictionnaire analogique de C. Maquet ${ }^{34}$, le Dictionnaire des idées suggérées par les mots de $\mathrm{P}$. Rouaix ${ }^{35}$, et le Nouveau Dictionnaire analogique du français de $\mathrm{D}$. et $\mathrm{D}$. Delas ${ }^{36}$ rendent tous trois des services similaires : à travers leur nomenclature organisée selon l'ordre alphabétique des mots-centres qu'ils retiennent, ils aident à se remémorer des mots oubliés ou ils font découvrir des mots inconnus. Ces ouvrages peuvent ainsi être principe de créativité et source d'enrichissement $\mathrm{du}$ vocabulaire.

Des dictionnaires de synonymes actuellement disponibles, deux peuvent fort utilement figurer dans la liste des ouvrages qu'on aura avantage à consulter : le Dictionnaire des synonymes de $\mathrm{H}$. Bénac ${ }^{37}$ et le Nouveau dictionnaire des synonymes de E. Genouvier, C. Désirat et T. Hordé ${ }^{38}$.

Le premier est un ouvrage classique ; conforme au Dictionnaire de l'Académie française du point de vue de la nomenclature et des descriptions lexicales, il propose des regroupements de synonymes dont il marque judicieusement les nuarices. Le second innove tant par rapport au précédent que par rapport à son aîné chez Larousse, le Dictionnaire des synonymes de $\mathrm{R}$. Bailly ${ }^{39}$.

32. Troisième édition, revue et corrigée, Paris, Larousse, 1972, XLIX-805 p.

33. Paris, Presses universitaires de France, 1975, XXXII-682 p.

34. Dictionnaire analogique. Répertoire moderne des mots par les idées, des idées par les mots, d'après les principes de P. Boissière, Paris, Larousse, 1971, VII-591 p.

35. Paris. Armand Colin. 31e édition. 1974. $538 \mathrm{p}$

36. (Paris), Hachette-Tchou, 1971, XXIV-609 p.

37. Paris, Hachette, 1956, (réédité en 1978), 1026 p.

38. Paris, Larousse, $1977,510 \mathrm{p}$.

39. Paris, Larousse, 1947, $626 \mathrm{p}$. 
Conçu à la façon du D.F.C., le Nouveau dictionnaire des synonymes est avant tout destiné au rédacteur ; chaque article est axé sur des exemples ; ainsi les synonymes y sont généralement replacés dans leur contexte et considérés en fonction de tel ou tel type d'emploi (Ex. : plus courant, plus général ; etc.).

En tout état de cause, les dictionnaires de synonymes ne sauraient dispenser du recours aux grands dictionnaires de langue dans lesquels on trouve par ailleurs un inventaire exhaustif des synonymes correspondant à chaque mot, lorsqu'il y a lieu.

Avec l'Encyclopédie du bon français dans l'usage contemporain de P. Dupré ${ }^{40}$, on est renvoyé aux dictionnaires de langue (Dictionnaire de l'Académie, Littré, Dictionnaire général de la langue française, Grand Larousse encyclopédique, Dictionnaire alphabétique et analogique de la langue française) et aux ouvrages de référence de nature grammaticale.

Cette encyclopédie, selon les termes mêmes de l'Introduction, a classé \& alphabétiquement les principales difficultés, subtilités, complexités, singularités du français d'aujourd'hui ${ }^{41} \gg$. Au sujet de chaque thème étudié, elle présente l'opinion des cinq dictionnaires mentionnés ci-dessus; suivent des extraits de grammaires, d'ouvrages de linguistique, de lexiques, de chroniques de langue, etc. ; enfin, l'équipe de rédaction prend position, soit sur l'ensemble du sujet traité, soit sur un point particulièrement controversé.

Travail de compilation soigné et bien présenté, cette encyclopédie est un excellent ouvrage de référence; en particulier, elle saura faire économiser du temps à quiconque écrit en français.

\section{LES GRAMMAIRES ET LES OUVRAGES SIMILAIRES}

Une logique rigoureuse interdirait de placer ici un tel titre. En effet, on a déjà présenté plusieurs sources d'information grammaticale. Qu'il suffise de rappeler que le Grand Larousse de la langue française offre un quasi-traité de grammaire, tant à l'intérieur des descriptions lexicales que dans les notices spécifiques de «grammaire et linguistique ».

Toutefois, reconnaitre que les renseignements de nature grammaticale ne sont pas que dans les grammaires, ce n'est pas nier pour autant que celles-ci doivent avoir place, comme telles, dans la liste des ouvrages de base en matière de langue française. Notre titre : "Les grammaires et les ouvrages similaires 》 a donc sa raison d'être.

Le traité classique auquel on accordera la place de choix au rayon des grammaires, c'est Le Bon Usage. Grammaire française avec des remarques sur la langue française d'aujourd'hui de Maurice Grevisse ${ }^{42}$.

Il existe bien sûr d'autres grammaires dont la structure et le vocabulaire tiennent mieux compte des recherches et des progrès de la linguistique moderne,

40. Paris, Editions de Trévise, 1972, 3 vol, LXIV-2716 p.

41. Ibid., \&Introduction », p. 7.

42. Gembloux, Duculot, Dixième édition revue, 1975, VIII-1322 p. 
mais pour qui exerce le métier d'écrire, Le Bon Usage de Grevisse est vraiment le trésor grammatical irremplaçable. On ne compte plus les témoignages autorisés en ce sens ; ainsi le grammairien Robert Le Bidois écrit : "Le Bon Usage est à mon avis la meilleure grammaire française que nous ayons actuellement $»$; et André Gide : «Grevisse répond à toutes les questions flottantes... 》; et Jacques Cellard, titulaire de la chronique de langage au journal Le Monde : "Le Bon Usage est la plus classique et certainement la plus consultée de nos grandes gramımaires ${ }^{43}$.

D'aucuns trouvent difficile la consultation de cet ouvrage ; en règle générale, une fréquentation quelque peu assidue permet de découvrir les points de repère utiles et les clefs du volumineux index qui complète $L e$ Bon Usage. À ceux qui regrettent que l'auteur ne tranche pas les questions délicates ou controversées, on dira volontiers que la richesse de l'ouvrage tient précisément au fait qu'il respecte l'usage et qu'il donne place aux témoins de la langue; de ce fait, il stimule la réflexion tout en demeurant un guide sûr.

On se doit de souligner ici que le rédacteur à la recherche du mot juste ou d'une précision grammaticale sera très bien servi en consultant Le Français correct $^{44}$ de M. Grevisse. Ce guide pratique mérite son nom ; en effet, grâce à un index fort détaillé, il renvoie à des paragraphes numérotés dont l'exposé clair et bref permet de trancher mille et une difficultés courantes.

Une autre grammaire que les utilisateurs d'un service de documentation trouveront utile de consulter est l'œuvre de Georges et Robert Le Bidois, Syntaxe $d u$ français moderne ${ }^{45}$ dont la deuxième édition, revue et complétée, remonte à 1967. Écrite à l'intention «d'esprits formés 》, cette syntaxe du français moderne présente une analyse des faits de langage et de la structure du français dont on mesurera l'intérêt et la portée en lisant les quelques titres suivants : L'article devant les titres d'ouvrages; L'article défini dans la langue actuelle; «Dont», dans la langue de nos jours; «Tant», au lieu de «autant»; Emplois réguliers de «parce que».

Parmi les ouvrages qui regroupent les difficultés courantes de la langue, sans toutefois atteindre l'exhaustivité des grammaires, on accordera une bonne place à A. V. Thomas, Dictionnaire des difficultés de la langue française ${ }^{46}$, à J. Hanse, Dictionnaire des difficultés grammaticales et lexicologiques ${ }^{47}$ et à A. Sève et J. Perrot, Ortho Vert, Dictionnaire orthographique et grammatical ${ }^{48}$.

Ces dictionnaires traitent, par ordre alphabétique, de problèmes linguistiques qui se posent particulièrement à ceux dont le métier est d'écrire. Ainsi, à côté d'un article consacré à l'accord $\mathrm{du}$ nom, le Dictionnaire des difficultés de la langue française nous enseigne que nonpareil s'écrit en un seul mot, mais qu'il faut écrire non-sens avec un trait d'union.

43. Attestations reproduites sur le second rabat de la jaquette de la dixième édition de l'ouvrage.

44. Gembloux, Duculot, 1973, 400 p.

45. G. Le Bidois et R. Le Bidois, Syntaxe du français moderne, Paris, Picard (c1967), 1971, tome premier, XX-560 p., tome deuxième, X-794 p.

46. Paris, Larousse, 1956, XI-435 p.

47. Bruxelles, Baude, 1949, 758 p.

48. Paris, Editions sociales, 1976, 638 p. 
Le Dictionnaire des difficultés grammaticales et lexicologiques traite des noms composés et de l'adverbe de négation non. Il accorde aussi place à nonante marquant ainsi sa préoccupation, constante à travers l'ouvrage, d'attirer l'attention sur les belgicismes. De ce fait, il sera moins utile ici.

Ces deux dictionnaires ont une visée essentiellement pratique ; ils tentent de répondre aux questions les plus variées que se posent quotidiennement ceux qui sont en contact avec la langue française ; ainsi, orthographe, synonymes, difficultés d'ordre grammatical, pléonasmes, etc. sont traités avec maîtrise au fil d'articles brefs et clairs.

Alors que les deux dictionnaires que l'on vient de mentionner sont structurés sur le même modèle, le Dictionnaire orthographique et grammatical livre plutôt sa richesse dans de vastes tableaux portant sur des catégories ou des points clés de la langue française : adjectifs, division des mots, participes passés, etc. Sous les autres entrées qui se suivent selon l'ordre alphabétique, les renseignements sont généralement très sommaires ou plus exactement trop sommaires (Ex. : diktat, mot allemand; drap : nm. Tailler en plein drap).

Un dernier titre devrait entrer dans une bibliographie consacrée aux ouvrages de base en matière de français, c'est L'Art de conjuguer. Dictionnaire des huit mille verbes usuels ${ }^{49}$. Ce Nouveau Bescherelle, d'un contenu fort simple et d'un format modeste, est un guide éminemment pratique pour qui a "le souci de s'exprimer avec pureté et correction dans le français du $\mathbf{X X}^{\mathrm{e}}$ siècle ${ }^{50} \gg$.

\section{CONCLUSION}

Quelques remarques s'imposent en guise de conclusion.

Il est d'abord utile de rappeler que l'on a eu le souci constant d'assurer que les titres proposés répondent au critère défini au départ; on a, bien sûr, délimité la notion de documentation de base en matière de langue française en visant un public particulier, celui des utilisateurs d'un service de documentation qui, à un titre ou à l'autre, exercent le métier de dire ou d'écrire le français aujourd'hui.

On reconnaîtra bien qu'en un domaine tel que le français d'aujourd'hui, il est difficile de tracer la ligne entre ce qu'on peut appeler les « ouvrages de base les « autres». C'est dire que d'aucuns, guidés par leur connaissance générale de la langue ou par leur expérience professionnelle, souhaiteront rayer certains titres proposés ou en ajouter de nouveaux.

Tous conviendront cependant que les instruments de recherche les plus précieux en soi ne livrent leurs richesses qu'au terme d'une patiente adaptation du lecteur. Aussi, croyons-nous que celui qui fréquentera les ouvrages mentionnés au cours du présent article y trouvera une base solide pour mieux connaître et pour mieux traiter la langue française.

49. Nouvelle édition entièrement remise à jour, Montréal, Hurtubise, 1972, 160 p.

50. Ibid., \& Avertissement 》, p. 2. 